\title{
Beam test of a very large area linear silicon drift detector
}

\author{
V.Bonvicini $^{c}$, L. Busso ${ }^{a}$, P.Giubellino ${ }^{a}$, M.Idzik ${ }^{a, 3}$, \\ A.Kolojvari ${ }^{a, 1}$, L.M. Montano ${ }^{a, 2}$, D. Nouais ${ }^{a}$, C. Petta ${ }^{b}$,

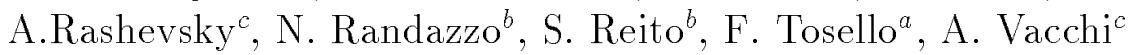 \\ ${ }^{a}$ INFN Torino, Italy, \\ ${ }^{b}$ INFN Catania, Italy \\ ${ }^{c}$ INFN Trieste, Italy
}

\begin{abstract}
We report the results of the first beam test of one of the very large area silicon drift detector prototypes produced for the ALICE Inner Tracking System, including data on efficiency, charge collection and linearity of the detector.
\end{abstract}

1) Also at Cyclotron Laboratory, St. Petersburg University, Russia 2) Also at CINVESTAV, MexicoCity, Mexico

3) Also at Fac. of Phys. and Nucl. Tech., Acad. of Mining and Met., Cracow, Poland 


\section{Introduction}

Since the original developments of the early eighties, Silicon Drift Detectors, SDD, have been the object of extensive study. Their good energy and spatial resolution make them a very attractive device for use in a number of applications. SDDs have been adopted to equip the two middle layers of the Inner Tracking System (ITS) of the ALICE experiment at the LHC [4]. The use of a large number of SDD in ALICE, more than 200 large area detectors, requires the assessment of large-scale production in industry with good reliability. As part of this program, originally carried out by the INFN DSI collaboration and now within the ALICE collaboration, prototypes of very large area linear silicon drift detectors, $8 \times 6.75 \mathrm{~cm}^{2}$, have been produced and tested in the laboratory.

The detectors are fabricated by Canberra Semiconductors N.V. (Belgium) on Neutron Transmutation Doped (NTD) 5" silicon wafers with a resistivity of $3 \mathrm{kOhm} \mathrm{cm}$. The total area is $6.75 \times 8 \mathrm{~cm}^{2}$, with a sensitive/total area ratio of $86 \%$. The detector has a "butterfly" bi-directional structure (see Fig. 1.1, 1.2 ) with a drift length of $32 \mathrm{~mm}$. On each half, the drifting charges are collected by an array of 384 anodes with a pitch of $200 \mu \mathrm{m}$. There are 265 cathode strips for each half per side (the side where the charge is collected and the opposite one, conventionally referred to as "anode" , or " $n$ ", side and " $p$ " side, respectively). The cathode pitch is $120 \mu \mathrm{m}$. All cathodes are connected to guard strips which run along the side of the detector, linking cathodes in the two opposite halves. The guard strips have a $40 \mu \mathrm{m}$ pitch, and are located alternatively on either side, in such a way that the potential difference between two contiguous guards is twice the one between two contiguous cathodes. The purpose of the guard strips is to progressively lower the negative high voltage of the cathodes down to ground, which is the potential of the edge bulk contacts. The high voltage resistive divider is integrated on the detector and is realised with $p+$ implantations. In addition, the last cathodes before the anodes, 8 on the anode side and 12 on the $\mathrm{p}$ side, are externally biased and constitute the so called "collection region".

\section{Setup and data taking conditions}

\subsection{Experimental setup and beam characteristics.}

Fig.2.1 shows the experimental apparatus installed at the 'T11' secondary beam of the CERN PS East Hall. X1,Y1, X2 and Y2 are scintillating counters, $1 \times 10 \times 0.5 \mathrm{~cm}^{3}$, that define the beam spot. They are aligned with two microstrip telescopes that measure particle trajectories. Each telescope is made of $61 \times 2 \mathrm{~cm}^{2}$ microstrip detectors, 3 for $x$ and 3 for $y$, having pitch of $50 \mu \mathrm{m}$. The SDD is placed between the two telescopes, and can be shifted in the $x-y$ plane with an accuracy of $\pm 5 \mu \mathrm{m}$. There are two other scintillating counters, not shown in Fig.2.1, one upstream and the other downstream, that can be used for time-of-flight measurement and contribute to the trigger during some runs. 


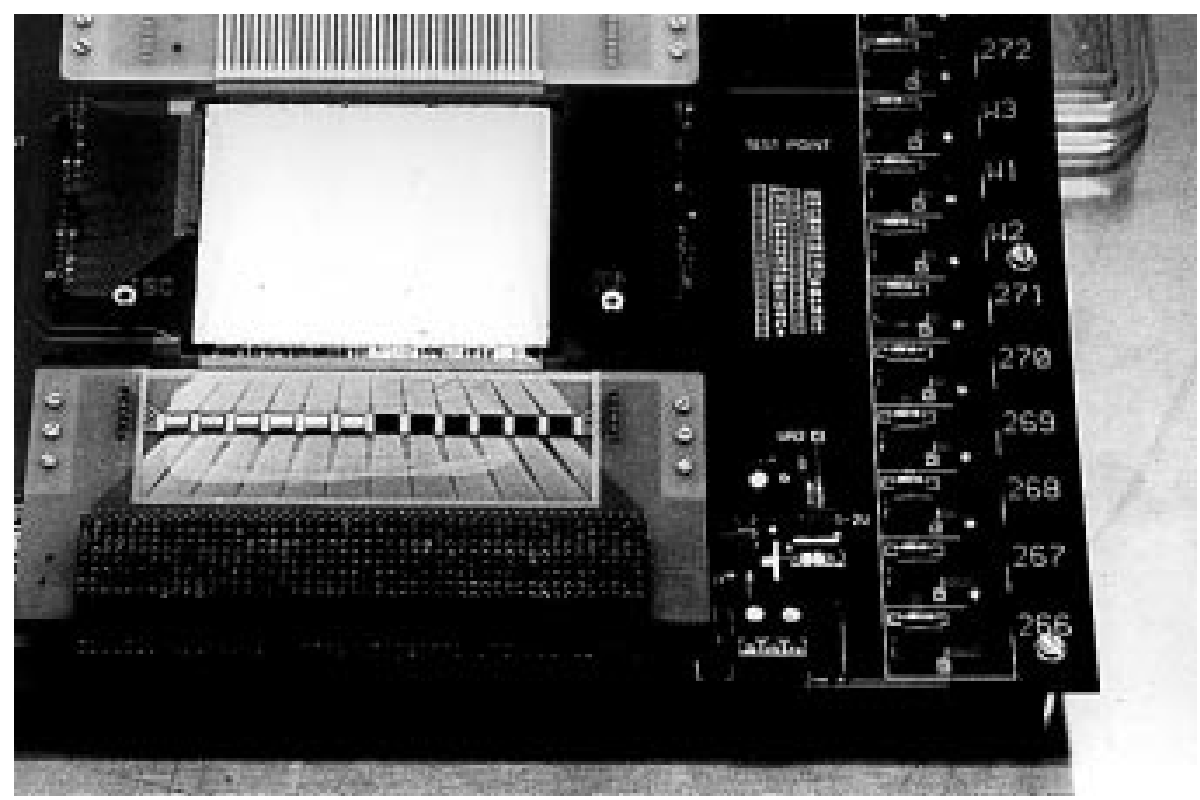

Figure 1.1. Photograph of the detector under test

Tab.2.1 sumarizes the characteristics of the T11 beam. Most of the time we used a $3 \mathrm{GeV} / \mathrm{c}$ negative beam, mostly $\pi^{-}$. For a group of run we used a $3 \mathrm{GeV} / \mathrm{c}$ beam of $62 \% \pi^{+}$and $32 \%$ protons (see Fig.2.2). The time structure of the beam consisted of one or two $300 \mathrm{~ms}$ extraction periods, depending on the PS supercycle, every $14.6 \mathrm{~s}$.

The electron mobility, and hence the drift velocity $\left(v_{d r i f t}=\mu \cdot \mathrm{E}\right)$, depends on temperature as $\mu \sim T^{-2.4}$. Therefore a temperature variation of $\approx 0.1^{\circ} \mathrm{C}$ corresponds, on average, to a change of the position of the reconstructed points of the order of $10 \mu \mathrm{m}$. Therefore, it is important to provide an accurate measurement and stabilization of the temperature and a continuous calibration of the drift velocity.

During the run, the temperature of the box containing the detector and the front-end electronics was monitored with a thermocouple, with a precision of \pm 0.2 degrees. The temperature reading was taken several times a day, and fairly large variations of up to 5 degrees were observed, and were linked to the variations of the ambient temperature. Unfortunately, given the way the setup was structured, the measured temperature is not trivially related to the temperature of the detector, and therefore cannot be used to correct the drift times, but only to provide guidance in the interpretation of the observed run-to-run variations of the drift velocity. 


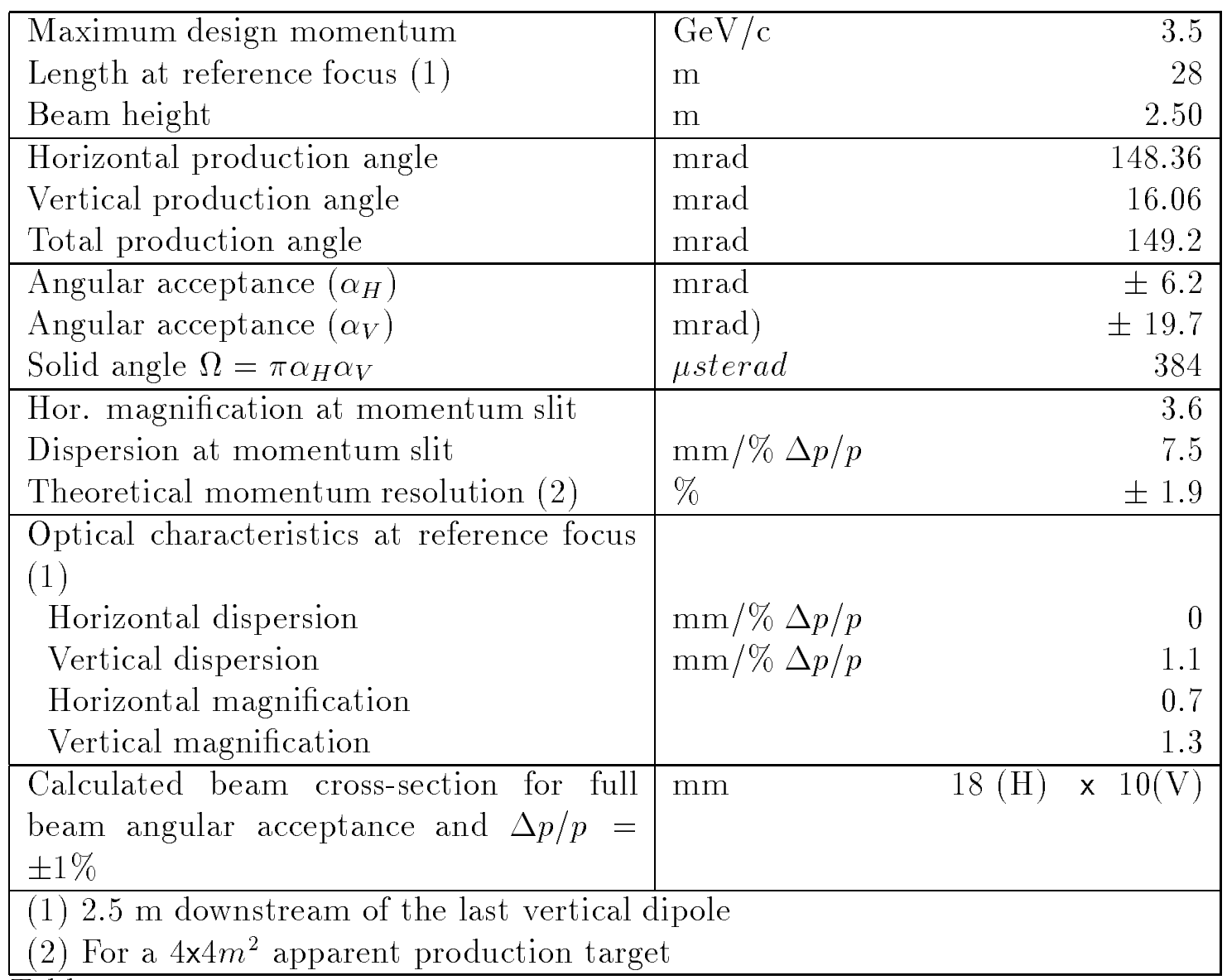

Table 2.1

Characteristics of the beam T11 


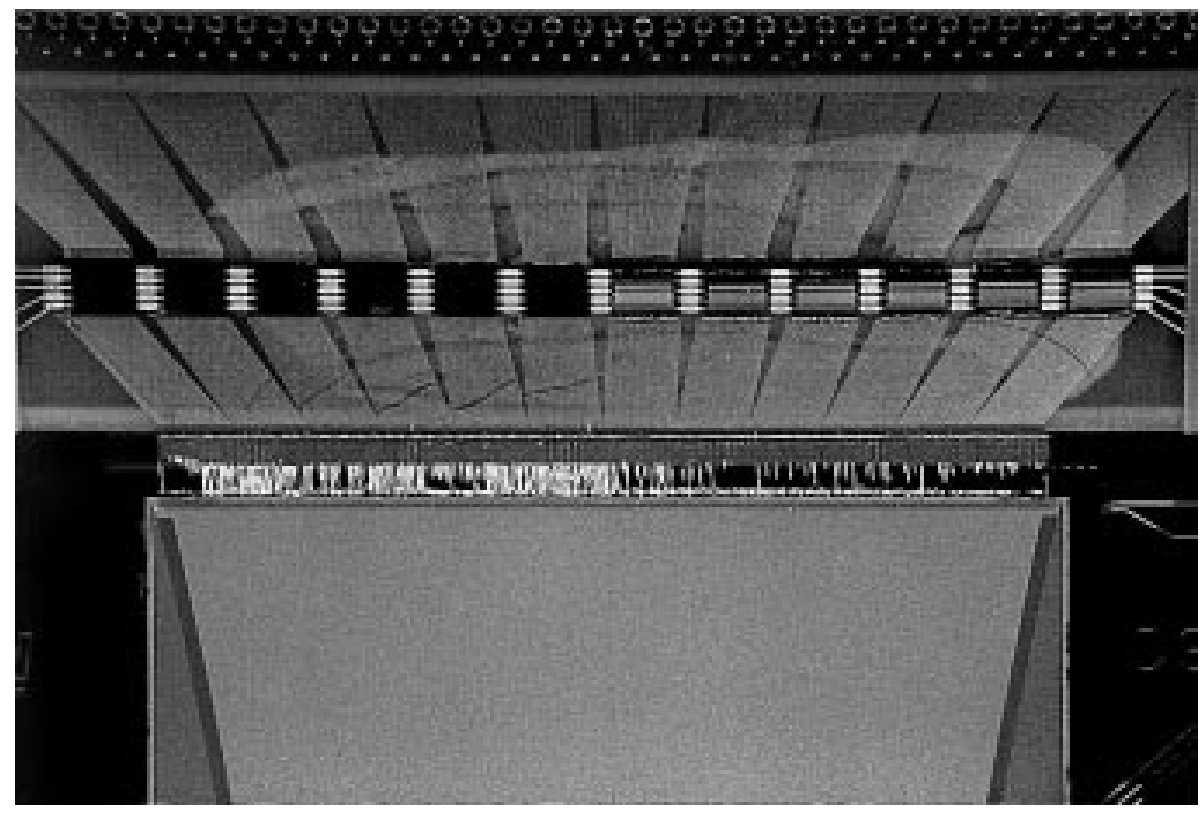

Figure 1.2. Photograph of the detector under test, detail of the anode region

\subsection{Front-end electronics and data acquisition.}

The readout chain of SDD includes a VLSI front-end, a voltage amplifier/driver and a Flash ADC system.

The front-end chip, OLA, is a very low noise 32-channel full-custom integrated circuit in bipolar technology [2]. Each channel features a charge sensitive preamplifier, a semi-gaussian shaper and a symmetrical line driver. The circuit sensitivity is $\sim 30 \mathrm{mV} / \mathrm{fC}$, the shaper peaking time is $55 \mathrm{~ns}$ for a $\delta$-like input signal, and the power consumption is $\sim 2.4 \mathrm{~mW} /$ channel. The Equivalent Noise Charge (ENC) was measured to be $\sim 230 e^{-}$at 0 detector capacitance. The linear dynamic range of the OLA chip is $\sim 12 f C$ for the positive and $\sim 16 \mathrm{fC}$ for the negative output. The latter was used in the setup for the present test.

The OLA output signals, through a $3 \mathrm{~m}$ long $50 \Omega$ coaxial cable, feed a voltage amplifier whose task is to drive a $34 \mathrm{~m}$ long, individually shielded, twisted pair cable up to the Flash ADCs. The voltage amplifier (Fig.2.3), originally developed for the gas drift chambers of the experiment PS201 [7] [8], has an input range of $0-350 \mathrm{mV}$ and a tunable gain, which was set at 5.6. The design is based on a NE5539 operational video amplifier with an input overcompensation that ensures good rise time response at the end of the twisted-pair cable.

The FADC system is the DL350 [9], developed by Laben and Dr.B.Struck for the PS201 experiment at LEAR. It is based on $100 \mathrm{MHz}, 8$ bit resolution, 


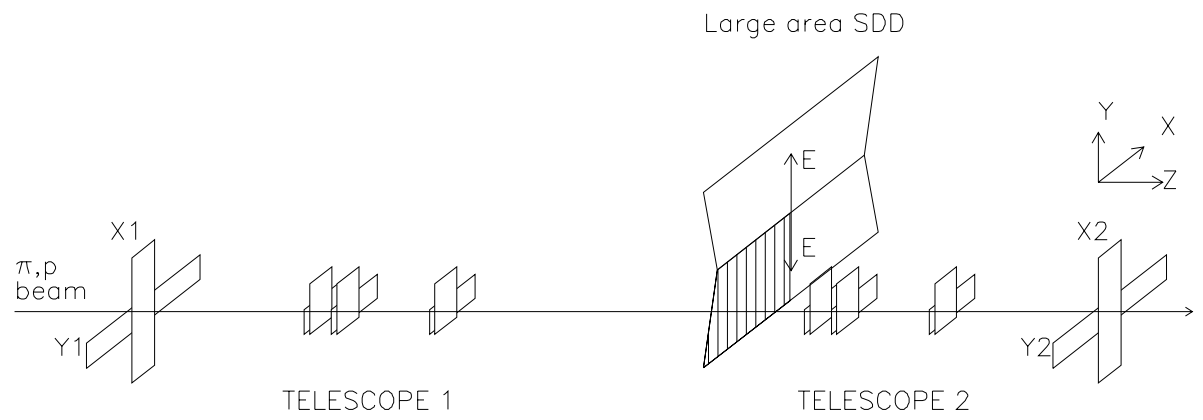

Figure 2.1. Experimental setup at the $T 11$ installation, not to scale. X1,Y1,X2, Y2 : trigger scintillators; Telescopes 1 and $2: 50 \mu \mathrm{m}$ pitch $(1 \times 2) \mathrm{cm}^{2}$ microstrip detectors; SDD: the two drift regions are shown, with the instrumented area hatched.

FADC chips having an input circuitry that expands the dynamic range to 10 bits. The transfer function is:

$F A D C_{\text {out }}=V_{\text {inp }} * 256 /\left(0.282+0.718 * V_{\text {inp }}\right)$

The input circuit has a bandwidth from DC to about $30 \mathrm{MHz}$ and allows for tuning of the baseline offset via a programmable 6 bit DAC whose dynamic range matches with the lower quarter of the FADC input range. Each FADC has a 256 byte ring memory. During data acquisition all FADC channels are driven by a sampling clock that can be programmed to be 100,50 or $25 \mathrm{MHz}$; accordingly, the maximum recorded drift time is $2.5,5.1$ or $10.2 \mu \mathrm{s}$.

The system is equipped with a programmable zero-suppression module that compares the FADC memory contents, channel by channel, with a given threshold. When the digitized data raise above the threshold for two contiguous time bins, they are copied into an output buffer until they go below the threshold again for two time bins. Moreover, up to 17 samples before the signal leading edge and 5 samples after the trailing edge can be stored in the output buffer. This allows for baseline monitoring and/or software threshold tuning in the offline signal processing. The system is operated in common stop mode.

The VME based data acquisition system, DAQ, is controlled by an HP743 workstation, acting as VME master, that programs and reads out the CAMAC modules (TDCs, ADCs, scalers and I/O registers) and the front-end modules of the microstrip telescopes (CRAMs V550 and V551B from CAEN). The DAQ program running in the master VME CPU, under HP-UX operating system, is the ALICE Test Beam DAQ [1]. It performs the following tasks: CAMAC and telescope readout, global buffering, run control and recording.

The data acquisition for the SDD is performed by an 68030-25 $\mathrm{MHz}$ based VME CPU (FIC-8323 from CES) running the OS9 operating system. 

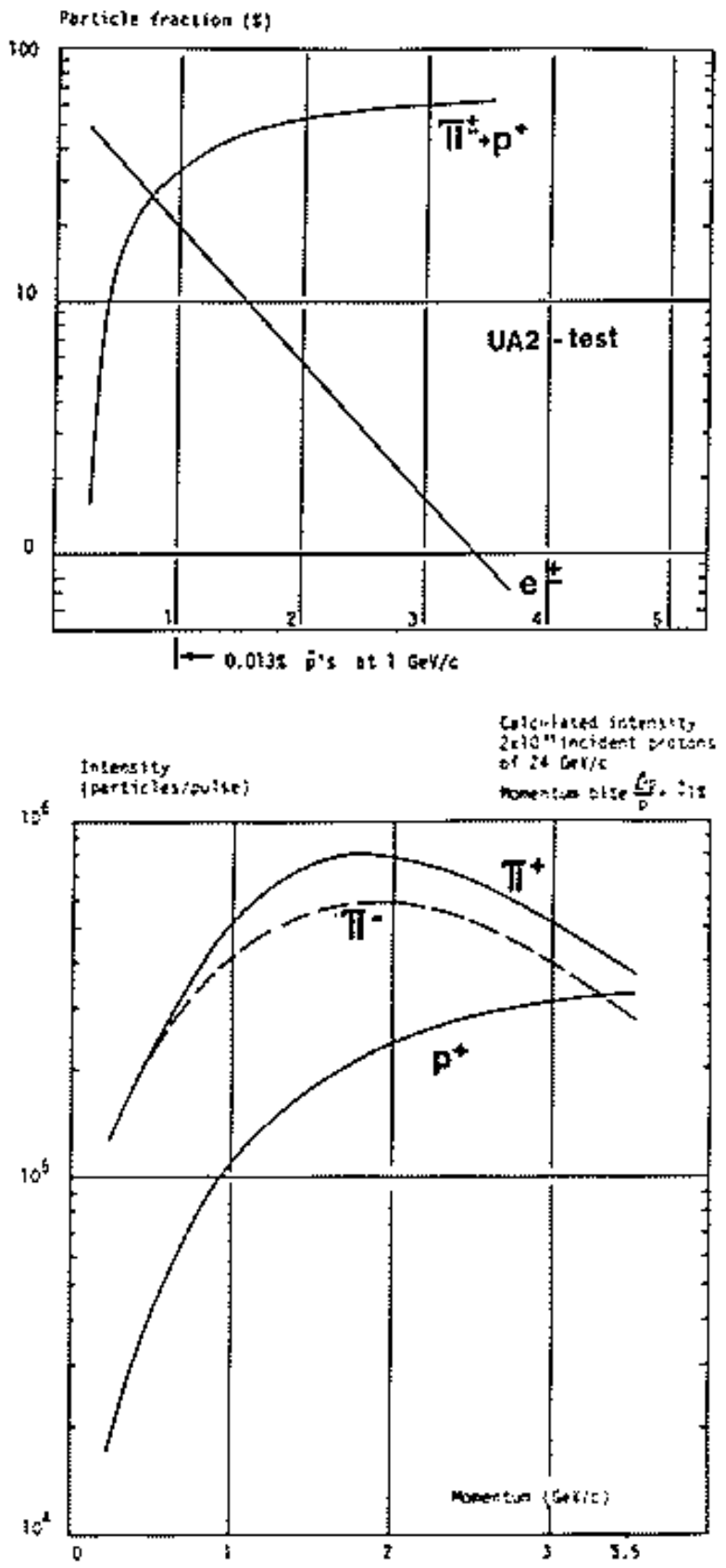

Figure 2.2. Observed particle fraction in the beam T11; upper part: UAD test (1987), particle identification by 2 Cerenkov counters +1 pre-shower detector; lower part: calculated intensity at the reference focus for $2 \times 10^{11}$ incident protons of $24 \mathrm{GeV} / \mathrm{c}$ in the primary beaam. 


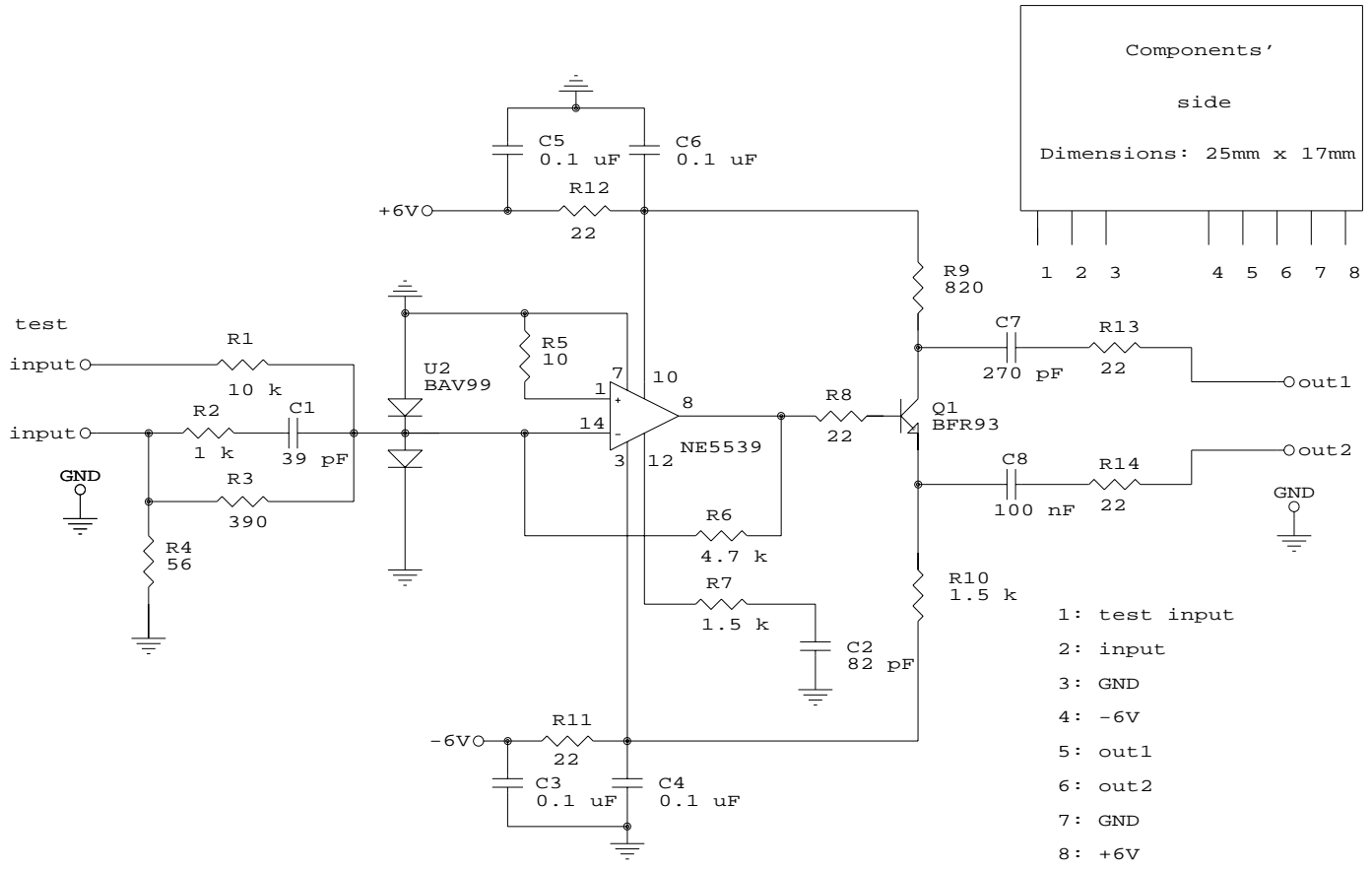

Figure 2.3. Voltage amplifier and twisted-pair cable driver

It is connected to the FADC crate via a differential VSB, for front-end programming and readout, and via a LAN to an HP735 workstation, for SDD run control and monitoring. The synchronization bewteen the two VME CPUs is implemented by a software handshake through fixed address memory words keeping the HP-743 as VME master all the time, to avoid bus error problems.

The readout dead time of the whole system is about $6 \mathrm{~ms}$; during the $300 \mathrm{~ms}$ extraction interval about 80 triggers given by the coincidence of the four scintillator counters, $\mathrm{X} 1{ }^{*} \mathrm{Y} 1{ }^{*} \mathrm{X} 2{ }^{*} \mathrm{Y} 2$, are collected and about 50 events are readout.

\subsection{Summary of data taken.}

The microstrip telescopes covered a $10 \times 10 \mathrm{~mm}^{2}$ area, on which the beam spot was centered. The SDD was equipped with 6 OLA chips, so it was possible to read out 192 anodes, i.e. about $4 \mathrm{~cm}$ along the anode direction for one side of the detector. In fact, only 96 anodes $(2 \mathrm{~cm})$, corresponding to 3 OLA chips, were read out during this data taking period to match the number of avaliable FADC channels. To scan the $33 \times 20 \mathrm{~mm}^{2}$ area which was read out, we have shifted the detector along both axis in steps of around $5 \mathrm{~mm}$.

Data have been mainly taken with a drift field of $460 \mathrm{~V} / \mathrm{cm}$ and using a FADC sampling frequency of $50 \mathrm{MHz}$. Fig. 2.4 shows the scanning of all the sensitive region of the detector obtained for these conditions.

The trapezoidal shape of Fig. 2.4 is due to the geometry of the sensitive 


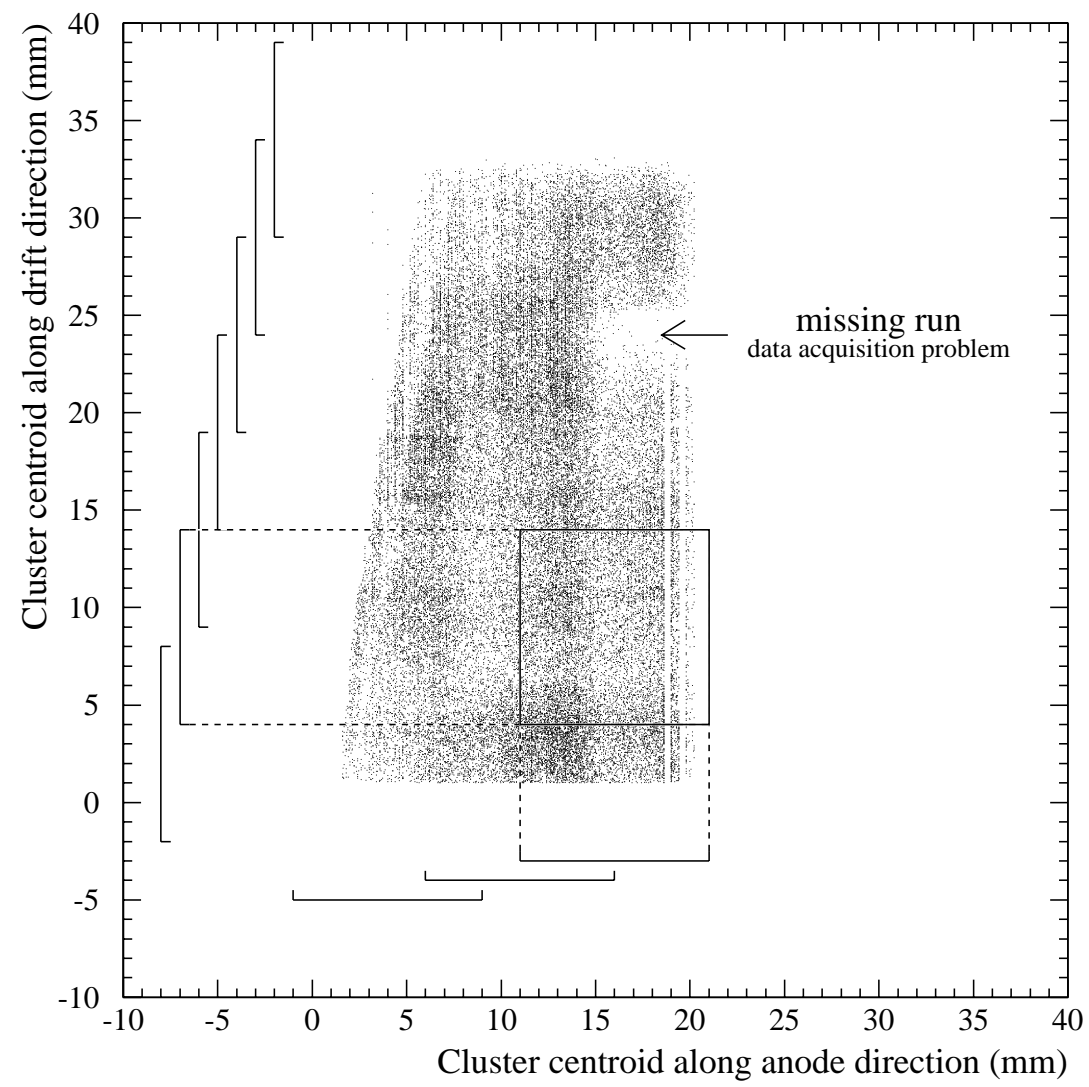

Figure 2.4. Cluster centroid position. All data taken with the drift field of $E=460 \mathrm{~V} / \mathrm{cm}$ and a sampling frequency of $50 \mathrm{MHz}$ are cumulated 
zone of the detector. The lower and upper limits correspond to the anode region and to the middle of the detector, i.e. the strip connected to the highest potential. The right boundary corresponds to the last anode connected to the electronics. We can observe empty strips which correspond to non working channels probably due to broken bonding between the anodes and the OLA chip. The left side corresponds to the guard strip zone.

Some data have also been taken at a lower field of $300 \mathrm{~V} / \mathrm{cm}$, and some more with a lower sampling frequency of $25 \mathrm{M} \mathrm{Hz}$. During the whole data taking period, for a fraction of the events the whole FADC memory was recorded (typically $10 \%$ to $20 \%$ ), while for the rest of the events the hardware zerosuppression was activated to reduce the data volume.

\subsection{Limitations from multiple scattering.}

The effects of multiple scattering in the SDD itself and in the telescope detectors represent an intrinsic limitation for the measurement of the spatial resolution of the SDD. A to evaluate this effect in one of the coordinates orthogonal to the beam direction. Tracks are generated starting from a random point in the first microstrip plane. The random directions of the tracks at their origin is chosen to intercept all the telescope detectors. At the intercept with a microstrip plane or with the SDD the tracks are tilted by a random scattering angle following a gaussian distribution with $\sigma$ evaluated from the known composition of the microstrip detectors and of SDD setup as listed in table 2.2

The positions of the SDD and of the telescope planes along the beam (known with an error of $1 \mathrm{~mm}$ ) are as listed in table 2.3

The standard deviations of the residual distributions with respect to the fitting straight line obtained with the six microstrip planes (see Fig. 2.5) are reported in table 2.4. In the last line is indicated the distance of the centroid of the SDD cluster to the fit. The MC does not include the SDD resolution, which of course contributes to the residual for the real data. The residuals on the SDD plane between the fitted track and the cluster coordinate for measured data are shown in fig. 2.6.

From these results it is only possible to conclude that the SDD resolution is compatible with the design figure of $30 \mu \mathrm{m}$, since the values which can be extracted $(37.8 \pm 13.8 \mu \mathrm{m}$ along the anode axis and $48.5 \pm 15.1 \mu \mathrm{m}$ along the drift time axis) are affected by very large systematic errors quite difficult to evaluate.

Similar results are obtained with a different Monte Carlo program [3] which does not take into account multiple scattering on the microstrip planes but only in the SDD setup ( $\mathrm{Si}$, plexiglass and G10). In this way it is obtained that the RMS in the SDD layer is 167.5 microns. Furthermore it is observed that taking into account multiple scattering in the strip layers, for example 


\begin{tabular}{|l|r|r|r|}
\hline$\mu$ strip Material & Thickness $[\mathrm{cm}]$ & Rad.length $[\mathrm{cm}]$ & Density $\left[\mathrm{g} / \mathrm{cm}^{3}\right]$ \\
\hline Si & 0.0300 & 9.36 & 2.33 \\
Mylar & 0.0180 & 28.70 & 1.39 \\
\hline Weighted radiation length & \multicolumn{2}{|c|}{$11.38 \mathrm{~cm}$} \\
$x / X_{0}$ & $4.22 \cdot 10^{-03}$ \\
Average m.s. plane angle for $\pi$ of $3.0 \mathrm{GeV} / \mathrm{c}$ (PS) & $0.234 \pm 0.026 \mathrm{mrad}$ \\
Average m.s. plane angle for $\pi$ of $375 \mathrm{GeV} / \mathrm{c}$ (SPS) & $0.0019 \pm 0.0002 \mathrm{mrad}$ \\
\hline SDD Material & Thickness $[\mathrm{cm}]$ & Rad.length $[\mathrm{cm}]$ & Density $\left[\mathrm{g} / \mathrm{cm}^{3}\right]$ \\
\hline Mylar & 0.0025 & 28.70 & 1.39 \\
Plexiglass & 0.1500 & 34.40 & 1.18 \\
Si & 0.0300 & 9.36 & 2.33 \\
G10 & 0.1600 & 19.40 & 1.70 \\
Plexiglass & 0.1500 & 34.40 & 1.18 \\
Mylar & 0.0025 & 28.70 & 1.39 \\
\hline Weighted radiation length & \multicolumn{2}{|c|}{$21.95 \mathrm{~cm}$} \\
$x / X_{0}$ & \multicolumn{2}{|c|}{$2.26 \cdot 10^{-02}$} \\
Average m.s. plane angle for $\pi$ of $3.0 \mathrm{GeV} / \mathrm{c}(\mathrm{PS})$ & $0.583 \pm 0.064 \mathrm{mrad}$ \\
Average m.s. plane angle for $\pi$ of $375 \mathrm{GeV} / \mathrm{c}(\mathrm{SPS})$ & $0.0047 \pm 0.0005 \mathrm{mrad}$ \\
\hline
\end{tabular}

Table 2.2

Material composition of the planes of microstrip detectors and of the SDD setup.

applying the Kalman filter method, does not improve significantly the result because the SDD factor of the multiple scattering is about 100 times larger (due to the material and to the lever arm) than the one for the strip telescope. Unfortunately mechanical constraints prevent the placement of the SDD close to the telescopes, so the only way to reliably measure the resolution of our detector is to use a much higher momentum beam. In table 2.4 are shown the residuals for a $350 \mathrm{GeV} / \mathrm{c}$ beam.

\section{Cluster finding method}

Typical FADC memory contents are shown in Fig.3.1 and Fig.3.2. They correspond to the signal collected on one anode in response to the passage of a particle near the center of the detector, and are representative of two series of runs: one with the SDD electric field of $\mathrm{E}=300 \mathrm{~V} / \mathrm{cm}$, and FADC sampling frequency $\mathrm{f}=50 \mathrm{MHz}$; the other to $\mathrm{E}=460 \mathrm{~V} / \mathrm{cm}$ and $\mathrm{f}=25 \mathrm{MHz}$.

The charge clusters are identified following a two-step procedure.

First, individual FADC outputs are analyzed. When the signal from the SDD is higher than a certain threshold level for two consecutive bins, it is considered as a "hit" and is used for the two-dimensional analysis. This first step was necessary for the treatment of non-zero-suppressed events, but was also 


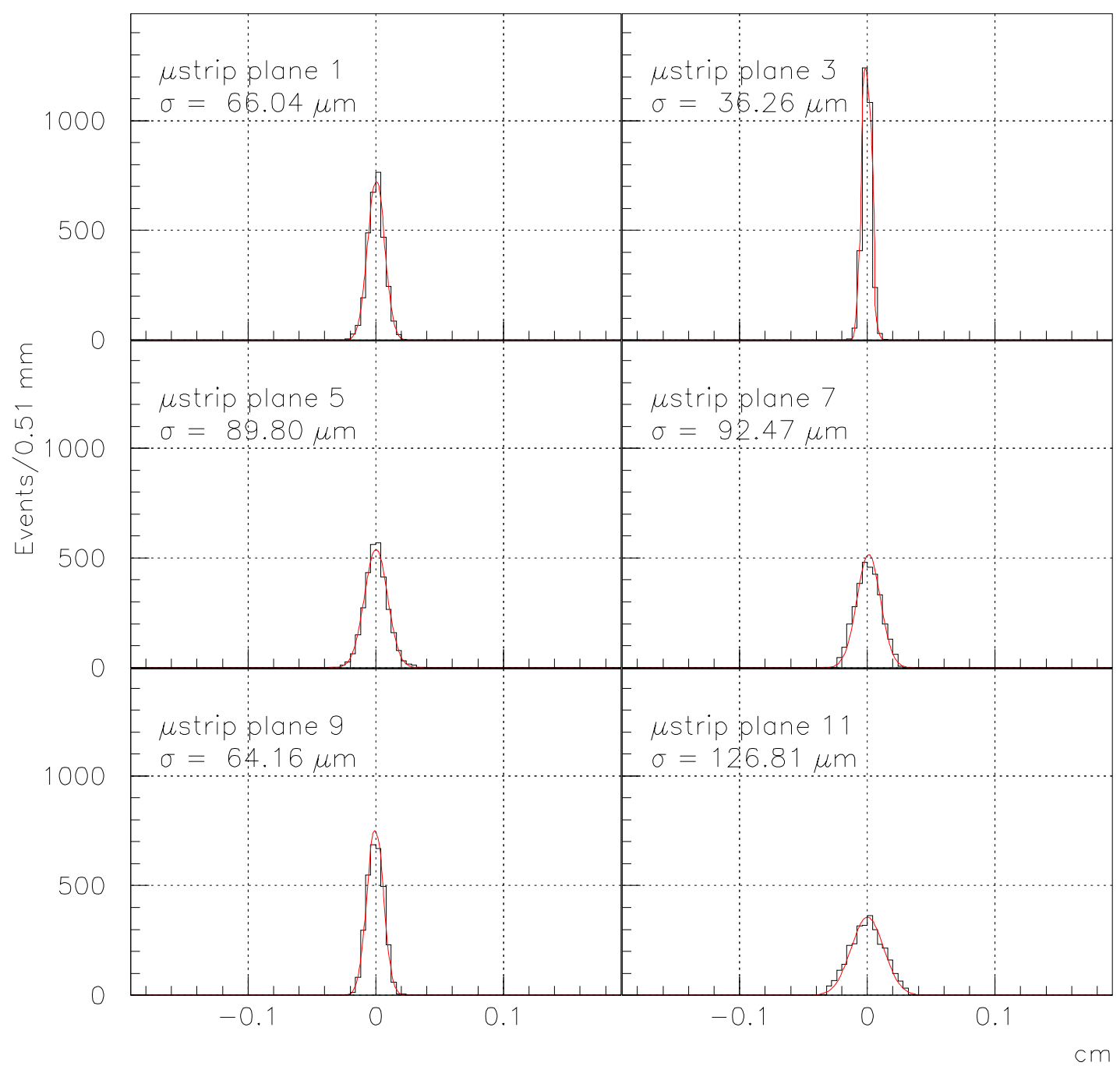

Figure 2.5. Residuals of microstrip hits to the fitting straight line from run 2300 . 

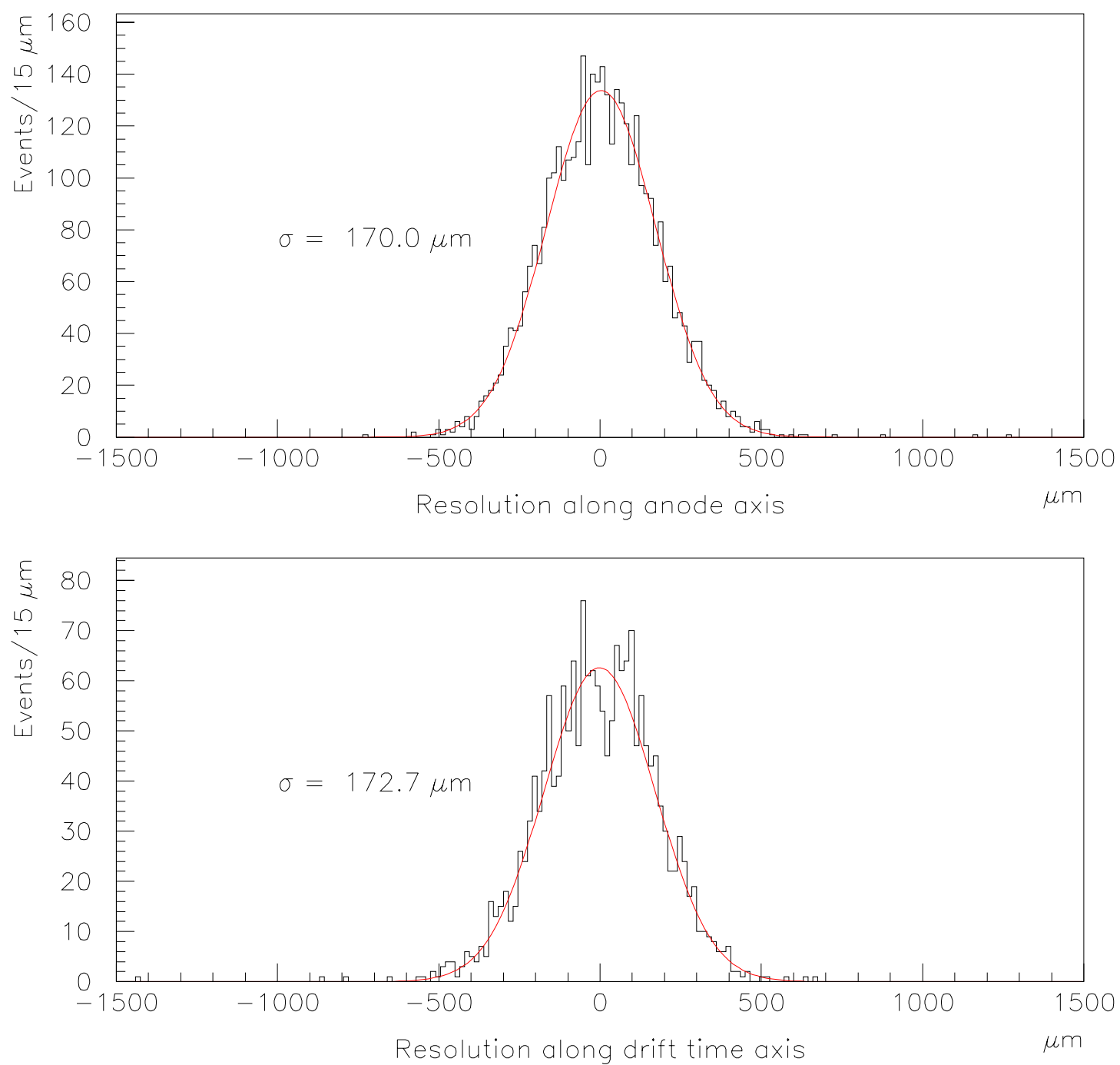

Figure 2.6. Residuals of SDD coordinates to the fitting straight line determined with microstrip telescopes (run 2300 ). 


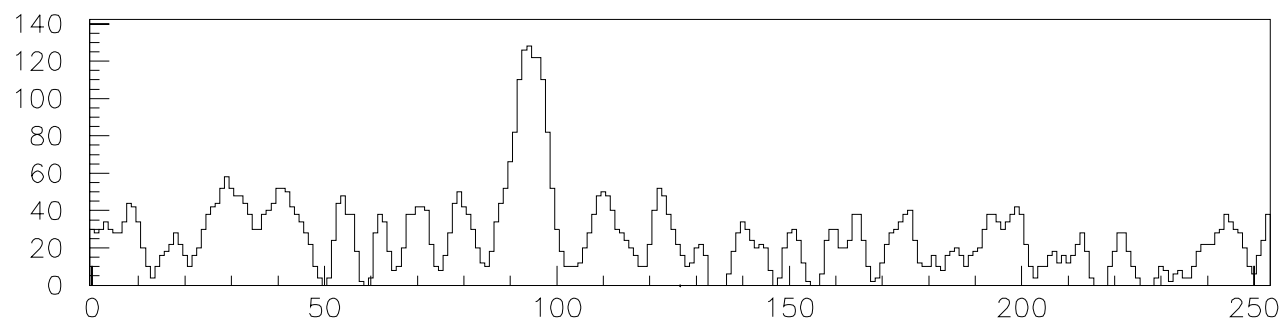

Figure 3.1. Typical FADC display for a particle crossing the middle of the SDD. Frequency $50 \mathrm{MHz}$. Electrical field $300 \mathrm{~V} / \mathrm{cm}$. Run \#2348.

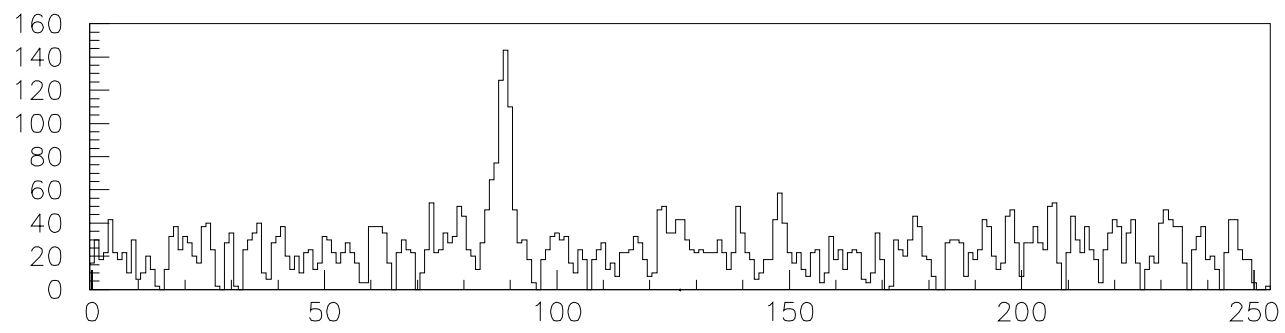

Figure 3.2. Typical FADC display for a particle crossing the middle of the SDD. Frequency $25 \mathrm{MHz}$. Electrical field $460 \mathrm{~V} / \mathrm{cm}$. Run \#2286. 


\begin{tabular}{|l|c|l|c|}
\hline \multicolumn{2}{|c|}{ Vertical planes } & \multicolumn{2}{c|}{ Horizontal planes } \\
\hline Plane \# & position [cm] & Plane \# & position $[\mathrm{cm}]$ \\
\hline 1$)$ & 0.0 & $2)$ & 1.7 \\
$3)$ & 8.4 & $4)$ & 10.1 \\
$5)$ & 38.8 & $6)$ & 40.5 \\
\hline SDD & \multicolumn{3}{|c|}{$137.4 \mathrm{~cm}$} \\
\hline 7$)$ & 155.2 & $8)$ & 156.9 \\
$9)$ & 163.6 & $10)$ & 165.3 \\
$11)$ & 194.0 & $12)$ & 195.7 \\
\hline
\end{tabular}

Table 2.3

Positions of SDD and of telescope planes along the beam; measurement error is $\pm 1 \mathrm{~mm}$.

\begin{tabular}{|l|r|r|r|}
\hline Plane \# & $\begin{array}{r}\text { Residuals from real } \\
\text { data (run 2300) }[\mu \mathrm{m}]\end{array}$ & $\begin{array}{r}\text { Residuals from M.C. } \\
\pi \text { of } 3 \mathrm{GeV} / \mathrm{c}[\mu \mathrm{m}]\end{array}$ & $\begin{array}{r}\text { Residuals from M.C. } \\
\pi \text { of } 365 \mathrm{GeV} / \mathrm{c}[\mu \mathrm{m}]\end{array}$ \\
\hline 1$)$ & $66.4 \pm 0.9$ & $56.56 \pm 0.18$ & $10.57 \pm 0.03$ \\
$3)$ & $36.3 \pm 0.5$ & $29.90 \pm 0.09$ & $10.80 \pm 0.03$ \\
$5)$ & $89.8 \pm 1.4$ & $78.55 \pm 0.25$ & $11.44 \pm 0.03$ \\
$7)$ & $92.5 \pm 1.0$ & $99.20 \pm 0.32$ & $11.04 \pm 0.02$ \\
$9)$ & $64.2 \pm 0.7$ & $62.41 \pm 0.20$ & $11.43 \pm 0.03$ \\
$11)$ & $126.8 \pm 1.5$ & $134.15 \pm 0.45$ & $10.23 \pm 0.03$ \\
\hline SDD anodes & $170.0 \pm 2.0$ & $165.74 \pm 0.53$ & $7.00 \pm 0.02$ \\
SDD drift time & $172.7 \pm 3.1$ & & \\
\hline
\end{tabular}

Table 2.4

Standard deviation of the residuals, on the SDD plane, between the fitting straight line the cluster coordinate for measured data.

applied to zero-suppressed events to provide the possibility of further adjusting the threshold level as compared to the one implemented in the hardware zero suppression module.

The second step takes the one-dimensional hits and combines them to make two-dimensional clusters. When hits in adjacent anodes overlap in time, they are considered as a part of a two-dimensional cluster. The final cluster is formed collecting all hits in adjacent anodes which overlap in time.

Once the cluster is defined, the charge integral and the position of the cluster center of gravity, with relevant errors and other variables corresponding to the particle-detector interaction, are calculated using all 1D-hits of the given 2D-cluster. 


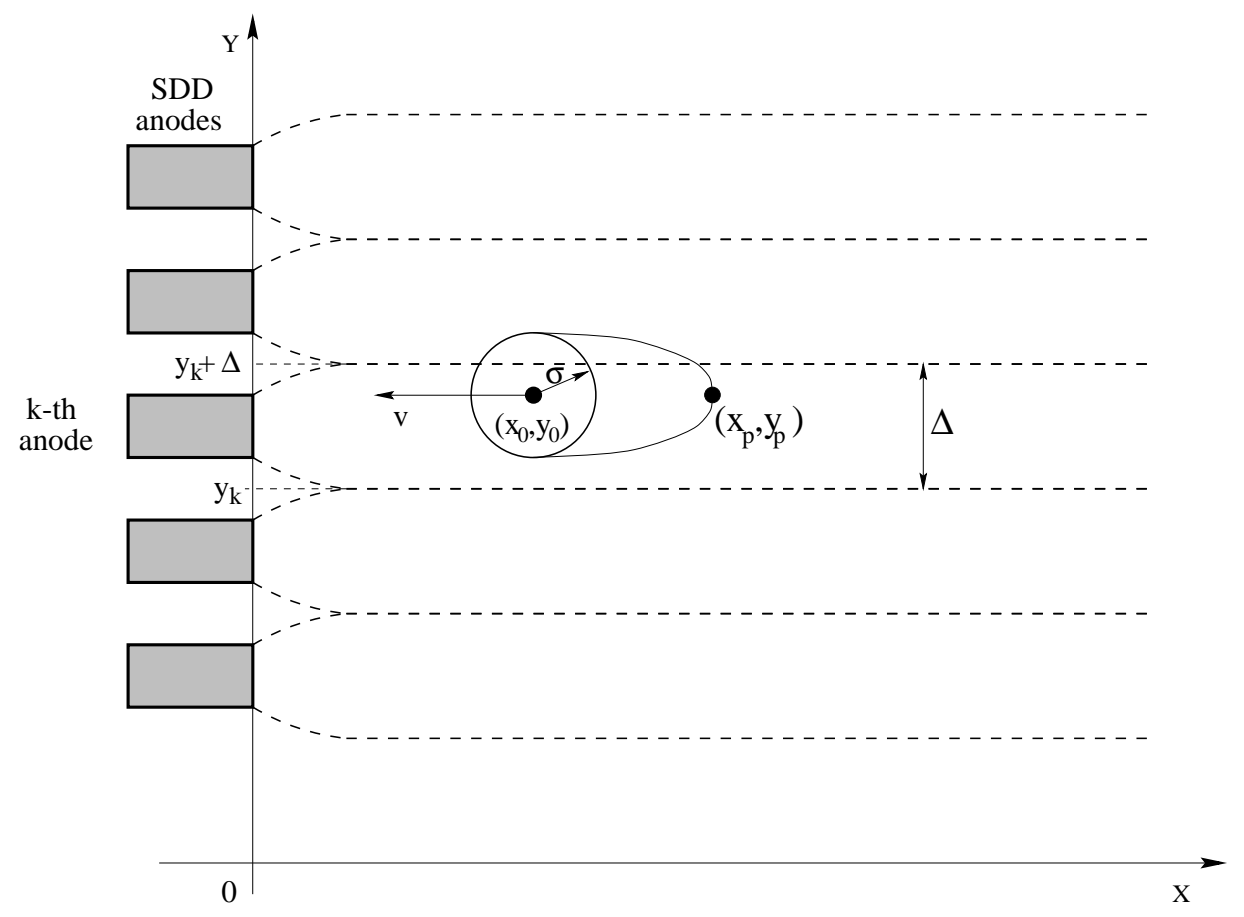

Figure 4.1. Coordinate system for SDD mathematical formalism and relevant variables.

\section{Results}

\subsection{Mathematical formalism}

For the description of the quantities experimentally measured with the SDD the following formalism, restricted to a two-dimensional model, is used.

Let's introduce a reference frame such that the X-axis is opposite to the drift direction and the $\mathrm{Y}$-axis is perpendicular to it. Let the "working edges" of the SDD anodes have $x=0$ (see Fig.4.1). At some moment $t$, the center of the charge cloud has coordinates $\left(x_{0}, y_{0}\right) ; \vec{v}$ is the drift velocity.

The density $\rho$ of the 2-D distribution of the charge cloud generated by a detected particle is approximated by Gaussian distribution:

$\rho(\vec{r}, t)=\frac{q}{2 \pi \sigma^{2}(t)} \cdot \exp \left(-\frac{\left(\vec{r}-\vec{r}_{0}(t)\right)^{2}}{2 \sigma^{2}(t)}\right)$

Here $\vec{r}=(x, y)$ and $\vec{r}_{0}=\left(x_{0}, y_{0}\right)=\vec{r}_{p}+\vec{v} t$ are the relevant 2 -D vectors for the $2-\mathrm{D}$ variable and for the position of the distribution center; $\vec{r}_{p}=\left(x_{p}, y_{p}\right)$ is the hit point, with $y_{p}=y_{0}$ since $\mathrm{X}$-axis is parallel to the drift direction; $\sigma$ is the gaussian parameter; $q$ is the total charge created in the SDD by a particle. 
Let an anode with number $k$ receive all the charge drifting in a SDD within the band of width $\Delta$ (Fig.4.1). The OLA amplifier has a peaking time of $55 \mathrm{~ns}$, the same order of magnitude of the expected input signal (standard deviation of 10-30 ns). Since the OLA amplifier has a linear response, the output voltage amplitude $u_{k}(t)$ is the convolution of the input current signal $i_{k}(t)$ coming from the detector by the response of the electronic chain to a $\delta$-like pulse, $h(t)$.

$u_{k}(t)=\int i_{k}(\tau) \cdot h(t-\tau) d \tau$

The current signal coming from the detector can be expressed as

$i_{k}(t)=v \cdot \int_{y_{k}}^{y_{k}+\Delta} d y \cdot \rho(\vec{r}=(0, y), t)$

where $v$ is the absolute value of the drift velocity $\vec{v}$.

During the data analysis the total charge $J$ associated to a cluster is calculated by summing the contents of the FADC memory locations within the area containing the charge cloud, after subtraction of the appropriate baselines.

$J=\sum_{k} \int d t \cdot u_{k}(t)$

Where the sum is calculated over the anodes involved.

Replacing $u_{k}(t)$ by the expression 4.2 we get:

$J=\sum_{k} \int\left(\int i_{k}(\tau) \cdot h(t-\tau) d \tau\right) \cdot d t$

The integral of a convolution is equal to the product of the integrals of each function since:

$$
\begin{aligned}
& J=\sum_{k} \int_{-\infty}^{+\infty}\left(\int_{-\infty}^{+\infty} i_{k}(\tau) \cdot h(t-\tau) d \tau\right) \cdot d t \\
& J=\sum_{k} \int_{-\infty}^{+\infty} i_{k}(\tau) \cdot\left(\int_{-\infty}^{+\infty} h(t-\tau) \cdot d t\right) d \tau \\
& J=\sum_{k} \int_{-\infty}^{+\infty} i_{k}(\tau) \cdot\left(\int_{-\infty}^{+\infty} h(T) \cdot d T\right) d \tau \\
& J=\sum_{k} \int_{-\infty}^{+\infty} i_{k}(\tau) \cdot d \tau \cdot \int_{-\infty}^{+\infty} h(T) \cdot d T
\end{aligned}
$$


Therefore, we can write $\mathrm{J}$ as:

$$
J=\sum_{k} \int_{-\infty}^{+\infty} h(t) \cdot d t \cdot \int_{-\infty}^{+\infty} i_{k}(t) \cdot d t
$$

where the first integral is a constant $K$ which depends only on the electronics characteristics and the second one is the charge collected on anode $k$

$J=K \cdot \sum_{k} q_{k}$

Finally it results that $J$ is proportional to the total charge $q$. Taking explicitly the function $\rho$ from (4.1) and assuming that $\sigma(t)$ varies slowly with time and can be considered as a constant during the integration over $t$ for $t \gg \sigma(t) / v$ (e.g. we neglect the diffusion process during the collection of the charge cloud by an anode, which should be true for the drift distances greater than $1-1.5 \mathrm{~mm}$ ), it is possible to calculate (4.4):

$J=K \cdot q$

From the diffusion equation it follows that

$\sigma^{2}(t)=D \cdot t+a$

Where $D$ is the diffusion coefficient of electrons in silicon and $a$ is a parameter describing initial dimension of the charge cloud. In this treatment we neglect the effect of Coulomb repulsion among the electrons.

After inserting (4.13) into (4.1), it is possible to obtain the following relation between the drift time $t$ and the maximum of the charge density:

$\rho_{\max }(t)=\frac{q}{2 \pi(D t+a)}$

Using the last relation we can derive an expression for the maximum signal amplitude obtained from the charge cloud. Suppose, that position of the charge distribution center $\overrightarrow{r_{0}}$ is within the band of width $\Delta$ collected by the anode $k$ (see Fig.4.1). Then, using (4.3) and (4.1) for $t_{0}=x_{p} / v$, we obtain:

$\max \left(u_{k}\right)=K v \cdot \frac{q}{2 \pi \sigma^{2}\left(t_{0}\right)} \cdot \int_{y_{k}}^{y_{k}+\Delta} d y \cdot \exp \left(-\frac{\left(y-y_{0}\right)^{2}}{2 \sigma^{2}\left(t_{0}\right)}\right)$

In the data analysis the distribution of $\max \left(u_{k}\right)$ was studied. Looking at (4.15) one can see that $\max \left(u_{k}\right)$ is a function of two random variables $q$ and $y_{0}$; the last one being uniformely distributed within interval $\left(y_{k}, y_{k}+\Delta\right)$. Introducing

$g=\frac{1}{\Delta} \int_{y_{k}}^{y_{k}+\Delta} d y \cdot \exp \left(-\frac{\left(y-y_{0}\right)^{2}}{2 \sigma^{2}\left(t_{0}\right)}\right)$ 


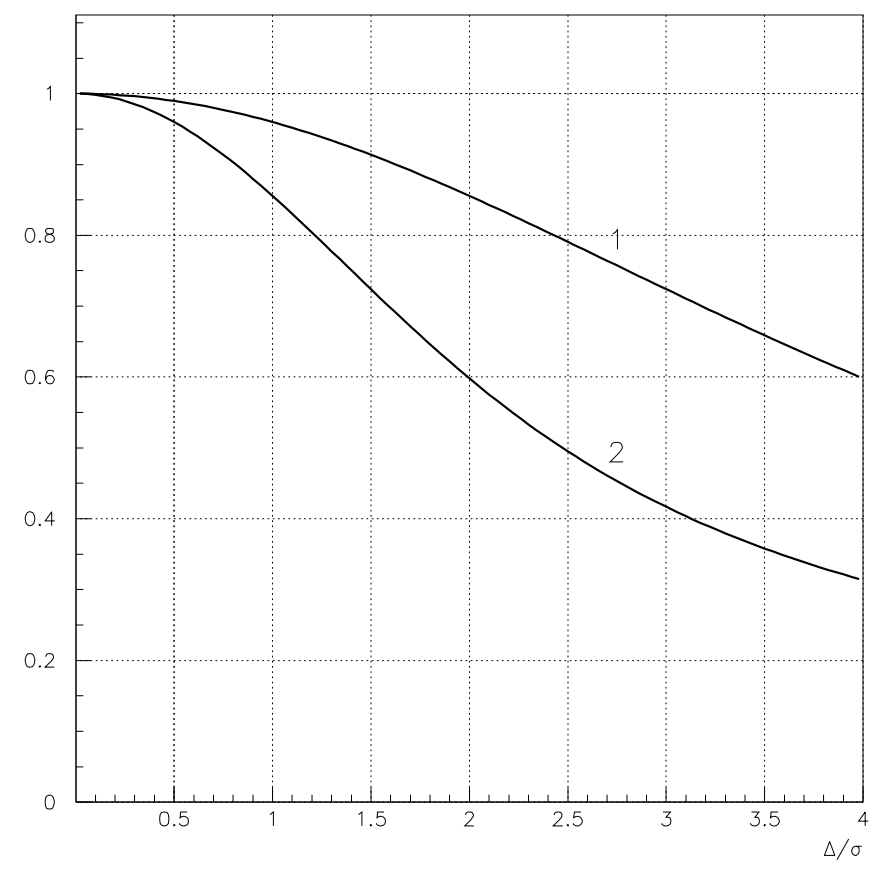

Figure 4.2. Dependence of the correction factor $g$ on the ratio $\Delta / \sigma$, for the case when $y_{0}$ is in the middle of the anode band $\Delta$ - curve 1 , and for the case with $y_{0}$ on the border of the band - curve 2 .

we can rewrite (4.15) as:

$\max \left(u_{k}\right)=K v \cdot \frac{q}{2 \pi \sigma^{2}\left(t_{0}\right)} \cdot \Delta \cdot g$

The factor $g$ depends on the ratio $\Delta / \sigma$ and on $y_{0}$. It reaches the maximum when $y_{0}$ is in the middle of the band $\Delta$, and minimum when it is on a border of the band, as shown in Fig.4.2.

It is seen that as far as

$\Delta / \sigma<1.0$

we may consider $g$ as a constant equal to 1 with $15 \%$ precision. Otherwise the following analysis is incorrect.

Supposing the (4.18) is true, which is a reasonable assumption for our case, we may neglect $g$ in (4.17) and obtain

$\max \left(u_{k}\right)=K v \cdot \frac{q}{2 \pi \sigma^{2}\left(t_{0}\right)} \cdot \Delta$ 
Inserting (4.13) into (4.19) we get:

$\max \left(u_{k}\right)=\frac{K v q \Delta}{2 \pi D} \cdot \frac{1}{t+a / D}$

In this case random value $\max \left(u_{k}\right)$ lineary depends only on one random variable $q$. The most probable value of $\max \left(u_{k}\right)$ should correspond to the most probable value of $q$ for $t$ given.

\subsection{Signal amplitude and diffusion}

Since the signal-noise discrimination is based on a threshold level, the signal amplitude is very important in determining the system efficiency. As we have seen, the signal amplitude depends on the amount of the charge deposited by the particle and on the drift time. In particular, the signal amplitude is inversely proportional to the drift time.

Therefore, if the drift time is large the signal can remain below the threshold leading to a reduction in the efficiency. This situation is illustrated in Fig.4.3, that shows distributions of two-dimensional clusters versus amplitude and drift time. The pictures are composed from different runs covering all the drift region, and the relative populations are not normalized. Parts corresponding to different runs are separated by vertical lines. The curve plotted is the average of the amplitude values. It is clearly seen that, at a field of $\mathrm{E}=300 \mathrm{~V} / \mathrm{cm}$ and starting from the middle of the drift region, the most probable value of the amplitude approaches the noise level and some of the events are lost. For $\mathrm{E}=460 \mathrm{~V} / \mathrm{cm}$ the signal is well separated from the noise level up to the largest drift times.

In Fig.4.4 is shown the same distribution of amplitudes vs. drift time for $\mathrm{E}=460 \mathrm{~V} / \mathrm{cm}$ and a $1 \mathrm{D}$ threshold of 30 units together with the fit of the most probable values of the cluster amplitudes. The units are FADC counts, which have a value close to one millivolt. The hyperbolic fit is quite good, as expected for the cluster amplitudes. It is interesting to note that in fig. 4.4(a) the noise band is absent at the same value of threshold as in Fig.4.3(a). This is probably due to the lower FADC sampling frequency $\mathrm{f}=25 \mathrm{MHz}$ in Fig.4.4(a) combined with the algorithm of comparison of signal and threshold. The hit is accepted only when at least two sequetial time-samples have amplitude above the threshold. Therefore those noise hits which are accepted as a signal at $\mathrm{f}=50 \mathrm{MHz}$, because they have two samples above the threshold, are rejected at $\mathrm{f}=25 \mathrm{MHz}$, because they have only one time-sample above the threshold.

We may try to compare eq. (4.20) with the results of our measurment, as shown in fig. 4.4, where is plotted the dependence of the most probable value of the cluster amplitude on the drift time with relevant hyperbolic fit parameters.

From fig. 4.5 we may obtain the most probable value of the integral of 


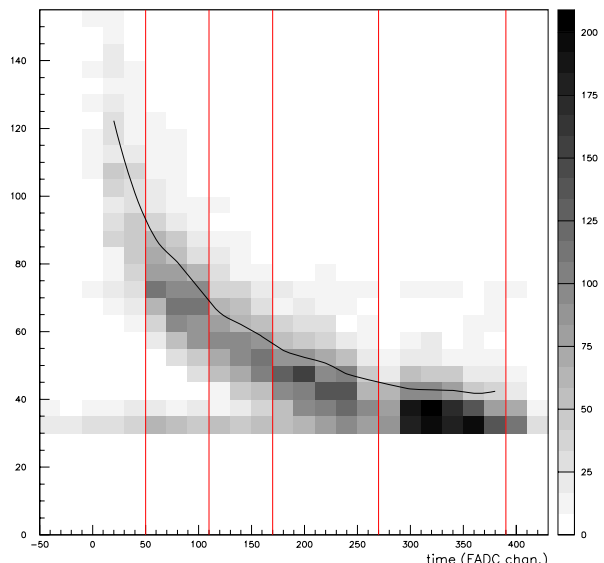

(a) $\mathrm{E}=300 \mathrm{~V} / \mathrm{cm} . \quad 1 \mathrm{D}$ threshold $=30 . \mathrm{f}=50 \mathrm{MHz}$

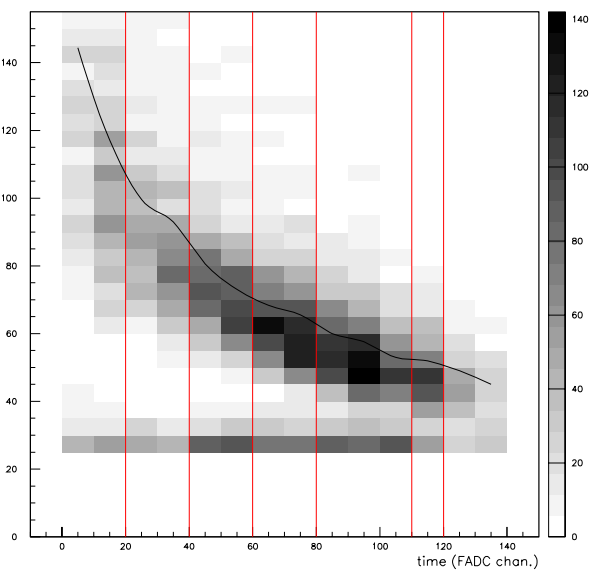

(b) $\mathrm{E}=460 \mathrm{~V} / \mathrm{cm} . \quad 1 \mathrm{D}$ threshold $=25 . \mathrm{f}=25 \mathrm{MHz}$

Figure 4.3. Distribution of clusters, amplitude versus drift time.

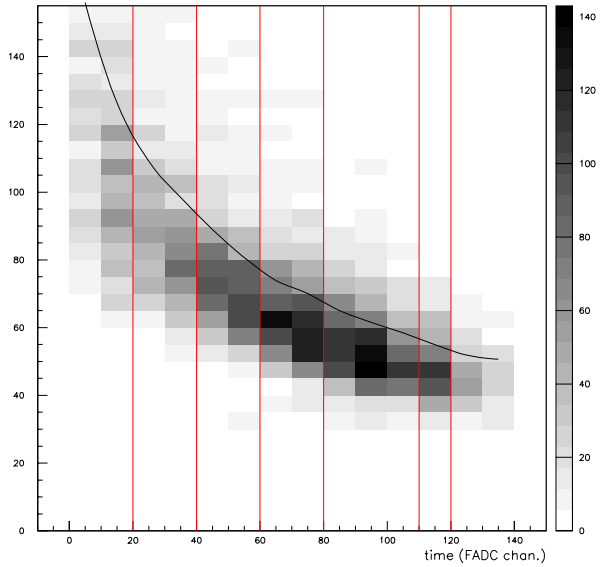

(a) $\mathrm{E}=460 \mathrm{~V} / \mathrm{cm} . \quad 1 \mathrm{D}$ threshold $=30$.

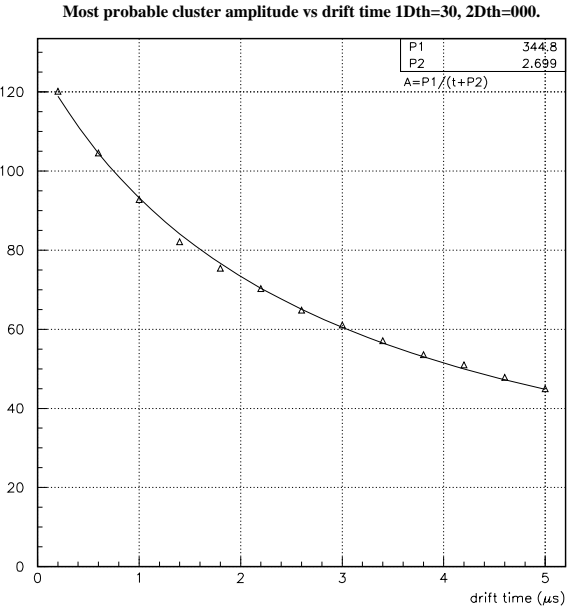

(b) Hyperbolic fit

Figure 4.4. Distribution of clusters versus amplitude and drift time. for $\mathrm{E}=460 \mathrm{~V} / \mathrm{cm}$ and $1 \mathrm{D}$ threshold $=30$ (the line reperesents the average of drift time bin). Hyperbolic fit of the MOST PROBABLE values of cluster amplitudes. 
charge:

$$
J=505 F A D C \text { counts } \times 40 \text { ns }=2.02 \cdot 10^{4} \text { FADC counts } \cdot n s
$$

where $505 F A D C$ counts is the average value of integral from fig. 4.5, which is multiplied by $40 \mathrm{~ns}$ - value of the FADC time bin, since this product was not performed by the data analysis software.

Now inserting $K$ from (4.12) into (4.20) we get:

$\max \left(u_{k}\right)=\frac{J v \Delta}{2 \pi D} \cdot \frac{1}{t+a / D}$

Using the experimentally measured drift velocity $v=6.1 \mu \mathrm{m} / \mathrm{ns}, \Delta=$ $200 \mu \mathrm{m}$ and the fitted parameters from fig. $4.4 P 1=\frac{\mathrm{Jv} \Delta}{2 \pi D}=344.8 \mathrm{bins} \cdot \mu \mathrm{s}$ and $P 2=a / D=2.699 \mu \mathrm{s}$, we calculate the diffusion coefficient $D$ :

$D=1.14 \cdot 10^{4}(\mu m)^{2} / \mu s ;$

Now it is possible to verify these results by checking condition (4.18). Using (4.23) and (4.13), we obtain that a $15 \%$ error is reached for drift times $t>0.82 \mu \mathrm{s}$. Therefore the two points at the left of the plot 4.4 do not satisfy the precision restriction. Therefore the values obtained for $D$ should be considered only as estimates. Since factor $g$ in (4.16) is systematically less then 1 , the obtained value $D$ should be biased. The unbiased value would be smaller.

\subsection{Charge transport and collection efficiency}

An analysis of the collection of the charge has been performed, using the subset of non zero suppressed data. In this way possible biases introduced by the hardware zero suppression could be avoided.

The following procedure was used to correctly measure the charge as a function of the drift time. An integration of the two-dimensional FADC spectrum was done within a rectangle of fixed dimensions. The center of the rectangle was chosen as the center of gravity of the two-dimensional cluster calculated during the formation of the two-dimensional clusters. If the rectangle is large enough, it should cover all the area occupied by the charge cloud and provide a correct evaluation of the charge. Dimensions of the rectangle for runs with $\mathrm{f}=25 \mathrm{MHz}$ and drift field $460 \mathrm{~V} / \mathrm{cm}$ were chosen as 6 anodes $\mathrm{x}$ 24 time bins. The scatter plot of the distribution of the cluster integral (i.e. the charge) versus the drift time, together with the plot of the average and most probable values of the cluster charge as a function of the drift time are presented in Fig.4.5. From the linear fit it follows that possible charge losses for the total drift region are compatible with zero within our errors.

\subsection{Signal to noise ratio.}

For the calculation of the total cluster charge it is better to choose an integration rectangle as large as possible, but on the other hand in this way one gets an increasing amount of noise integrated together with the signal. To 


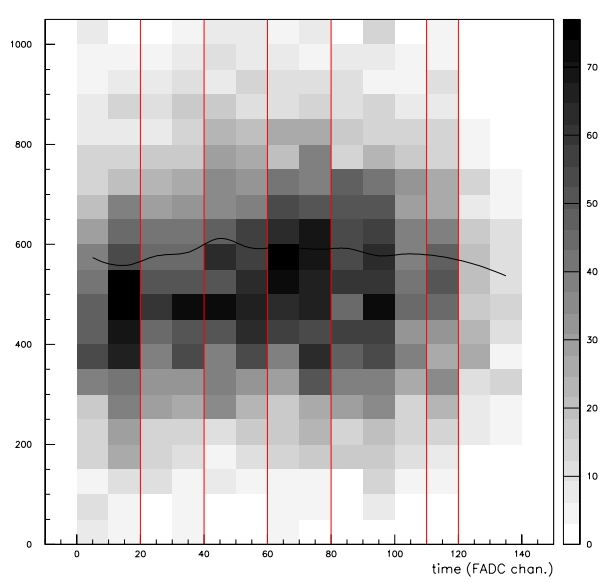

(a) Distribution versus charge and drift time.

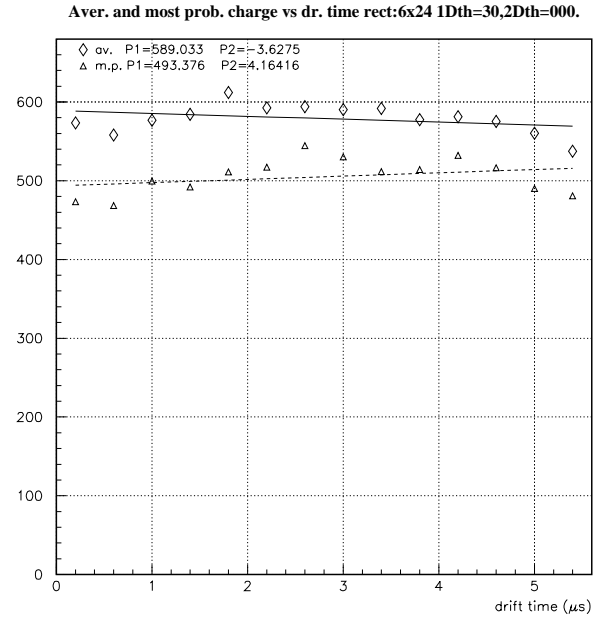

(b) Average and most probable charge vs drift time

Figure 4.5. Charge collection plots. for $\mathrm{E}=460 \mathrm{~V} / \mathrm{cm}$ and $1 \mathrm{D}$ threshold $=30$.

estimate the best possible signal to noise ratio, the average value of cluster integral calculated over a rectangle was divided by the RMS of the noise integral distribution. In this case, the noise distribution is defined as the distribution of the values of the integral calculated over a rectangle of the same dimensions used for the signal in the absence of signals generated by particles. The dependence of the signal to noise ratio on the dimensions of the rectangle was studied. For a drift field $\mathrm{E}=460 \mathrm{~V} / \mathrm{cm}$, the optimal rectangle was found to be of 3 anodes $x 3$ timebins. The dependence of this ratio on the drift time is presented in Fig.4.6.

\subsection{Detector efficiency}

We define the efficiency of the detector as the ratio between the number of tracks for which a cluster is detected in the SDD and the total number of reconstructed tracks. On the top of fig. 4.7, the efficency is represented versus the drift path for both values of the electric field $300 \mathrm{~V} / \mathrm{cm}$ and $460 \mathrm{~V} / \mathrm{cm}$. For the highest field, we observe an excellent efficiency practically up to the region farthest from the anodes. On the bottom of fig. 4.7, the inefficiency is shown with a zoomed scale. We see that up to $25 \mathrm{~mm}$ we are able to detect essentially all clusters. It has been previously demonstrated that for a given charge deposit, the amplitude of the signal decreases hyperbolically with the drift time due to diffusion effects. So, the probability that the amplitude does not reach the first threshold increases with the path length. The loss of amplitude is more pronounced for the lowest drift field because the velocity is 
charge/noise vs drift time. thresholds:1D=30,2D=000. rect.:3x3.

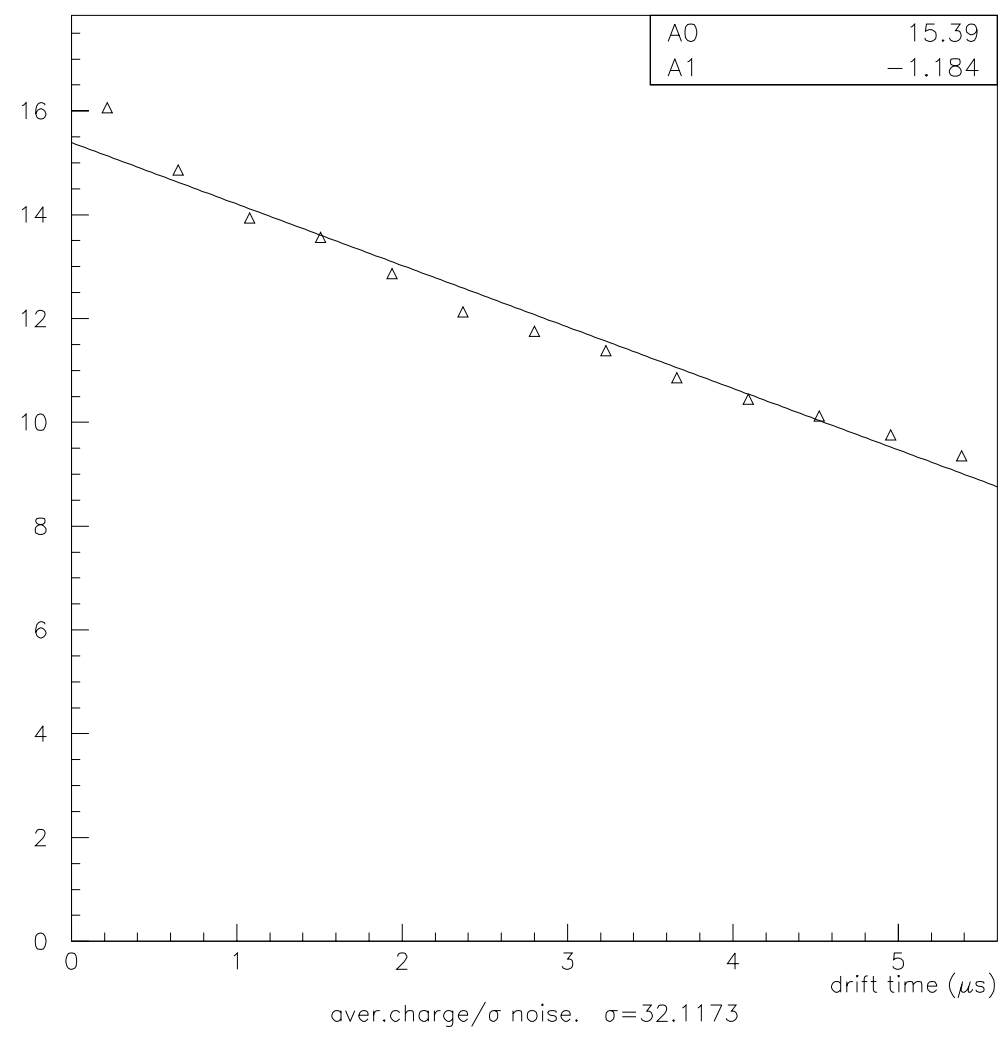

Figure 4.6. Ratio of the average charge to the RMS of the noise for $3 \times 3$ integration rectangle as a function of the drift time. 
smaller and therefore the drift time is larger for the same drift length. At low field the efficiency is very good only on the first centimeter.

\subsection{Detector linearity}

\subsubsection{Along the drift direction}

The linearity is evaluated by plotting the electron drift time in the SDD versus the corresponding coordinate of the projection of the track reconstructed using the microstrip telescopes. Fig. 4.8 shows the linearity curves for both values of the drift field, $E=300 \mathrm{~V} / \mathrm{cm}$ and $E=460 \mathrm{~V} / \mathrm{cm}$.

Since the microstrip planes cover only $1 \mathrm{~cm}$ of the drift region, the data taken successively at different positions are cumulated for each curve. For both fields, the detector presents a good linearity. The drift velocities, extracted from the slope of these curves, are equal to $v=4.0 \pm 0.02 \mu \mathrm{m} / \mathrm{ns}$ and $v=$ $6.1 \pm 0.03 \mu \mathrm{m} / \mathrm{ns}$ respectively for the lowest and the highest fields respectively.

The deviation between the position evaluated from the SDD and the reference position between the microstrip telescopes is shown in fig. 4.9. In addition to the dispersion of the clusters due to the finite resolution and multiple scattering, we observe systematic deviations along the drift path.

The drift velocity depends on the temperature through the mobility. This parameter is very sensitive to the temperature, usualy given by the expression

$$
\mu \propto T^{-2.4}
$$

In order to take into account these variations, we have evaluated the drift velocity for each run. In this way, the position deviation (fig. 4.10) decreases strongly and becomes lower than $50 \mu \mathrm{m}$ full width.

\subsubsection{Along the anode direction}

Fig. 4.11 shows the cluster centroid position along the anode direction versus the reference position obtained using the microstrip telescopes. This plot has a large scatter due to the resolution but the linearity remains good up to $3 \mathrm{~mm}$ from the side of the sensitive region, where the voltage divider region is.

The difference between the position measured by the SDD and that projected from the reference telescopes is plotted in fig. 4.12, versus the projected position, for three different mean drift paths: $9 \pm 5 \mathrm{~mm}, 19 \pm 5 \mathrm{~mm}$ and $29 \pm 5 \mathrm{~mm}$. In all situations, a non linearity, possibly due to the presence of the voltage divider, clearly appears at the left side of each curve. Excluding these zones, we see the maximum of fluctuations increasing from $50 \mu \mathrm{m}$ for the data close to the anodes to $200 \mu \mathrm{m}$ for the data far from the anode region. A small undesirable lateral electric field component could explain such variations on the position evaluated by the SDD.

\section{Acknowledgements}

The help and support of L. Riccati is gratefully aknowledged. We would like to express our thanks to B. Nielsen, A. Rivetti and V. Russo for comments 
and useful discussions, and to L. Simonetti and F. Rotondo for the essential technical help.

\section{REFERENCES}

1. H. Beker, A Portable Data Acquisition System.

2. W.Dabrowski et al., Nucl. Phys. B(PS) 44, (1995), 637.

3. B.Batiounia, private communication.

4. ALICE collaboration, CERN/LHCC, 95/71

5. P.Rehak et al, Nucl. Instrum. and Methods, A235, (1985), 224-234

6. A.Vacchi et al, Nucl. Instrum. and Methods, A306, (1991), 187-193

7. R.Armenteros et al., CERN/PSSC/86-4, PS201 Proposal

8. A.Adamo et al., Sov.J.Nucl.Phys. 55 (1992) 1732

9. F.Balestra et al., Nucl.Instr. and Meth. A323 (1992) 523

10. STAR note 170, 1994. 

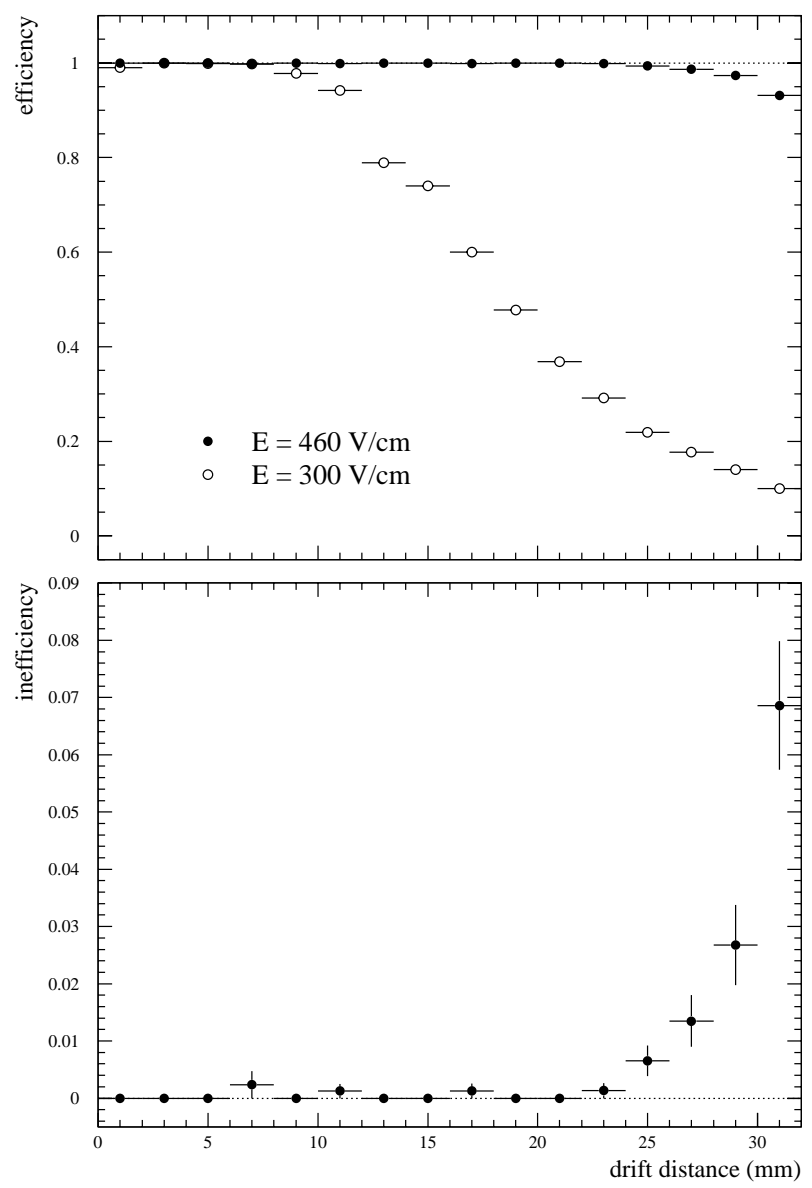

Figure 4.7. Top: Cluster finding efficiency versus the drift distance for two drift field: $E=300 \mathrm{~V} / \mathrm{cm}$ and $E=460 \mathrm{~V} / \mathrm{cm}$. Bottom: inefficiency versus the drift distance for the highest drift field. 


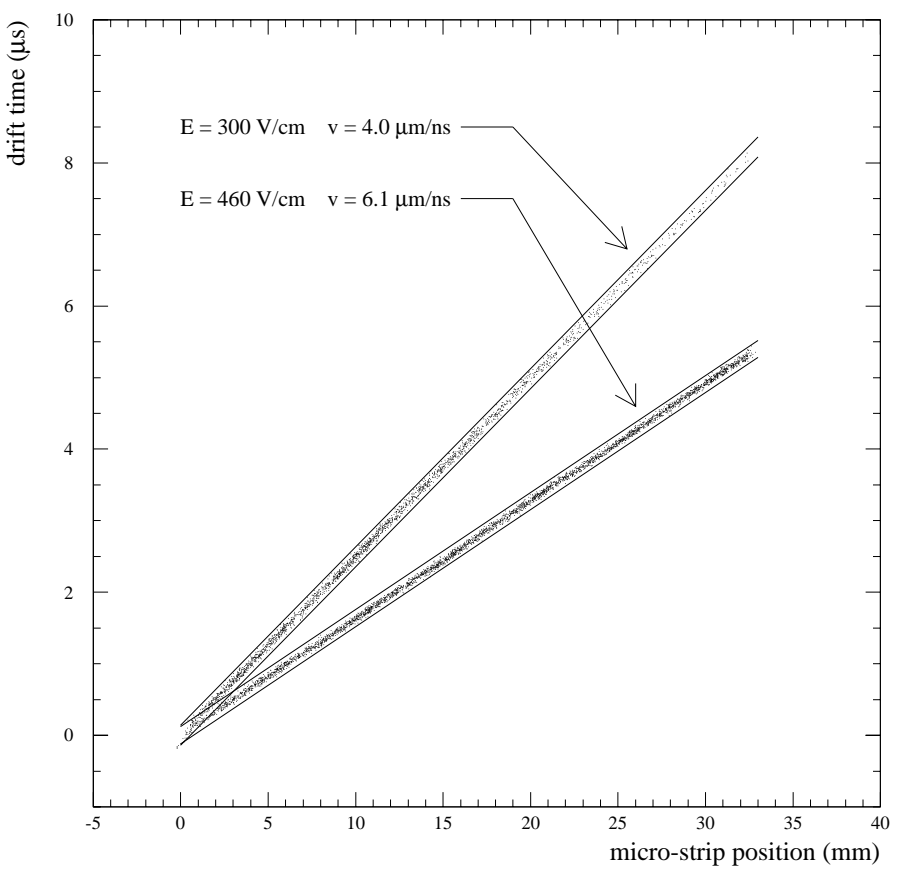

Figure 4.8. Drift time versus corresponding coordinate of the track projection 

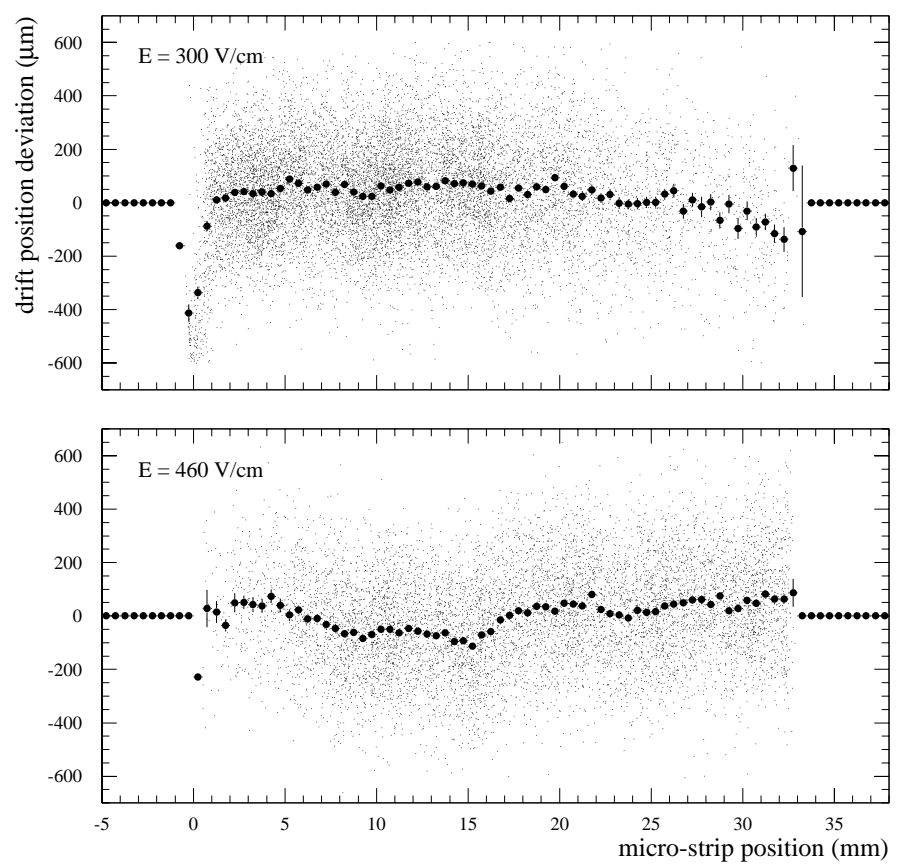

Figure 4.9. Drift linearity deviation versus corresponding coordinate of the track projection. a) $E=300 \mathrm{~V} / \mathrm{cm}$ and $b) E=460 \mathrm{~V} / \mathrm{cm}$. The mean drift velocity is considered. 

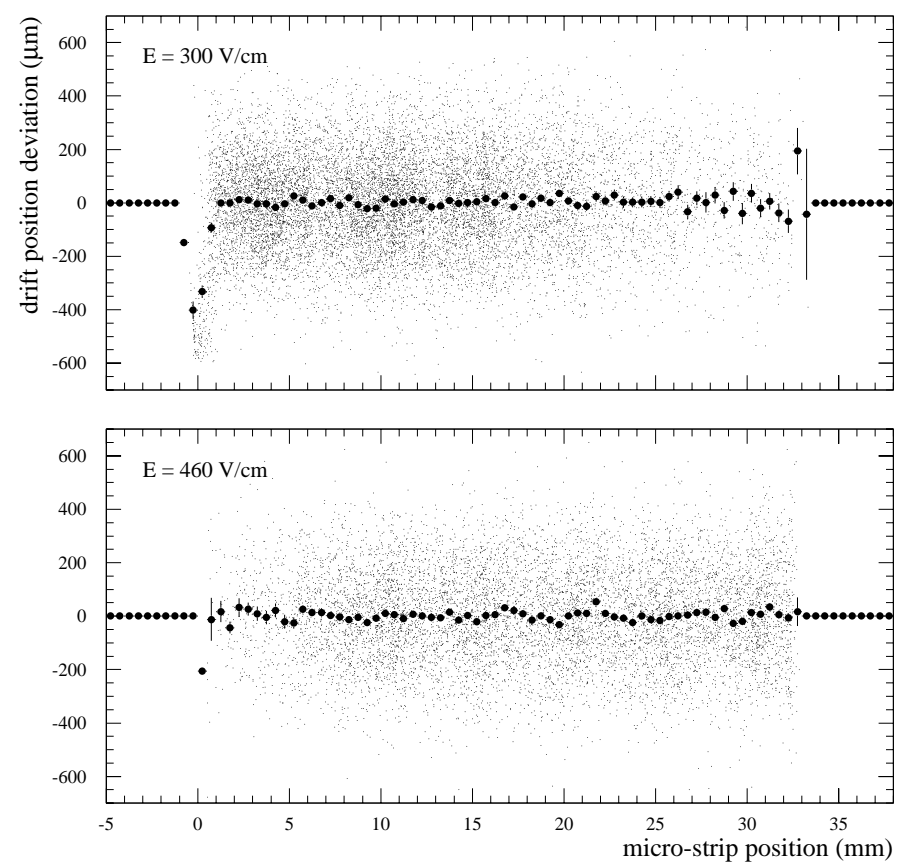

Figure 4.10. Same as fig. 4.9 with drift velocity evaluated independently for each run. 


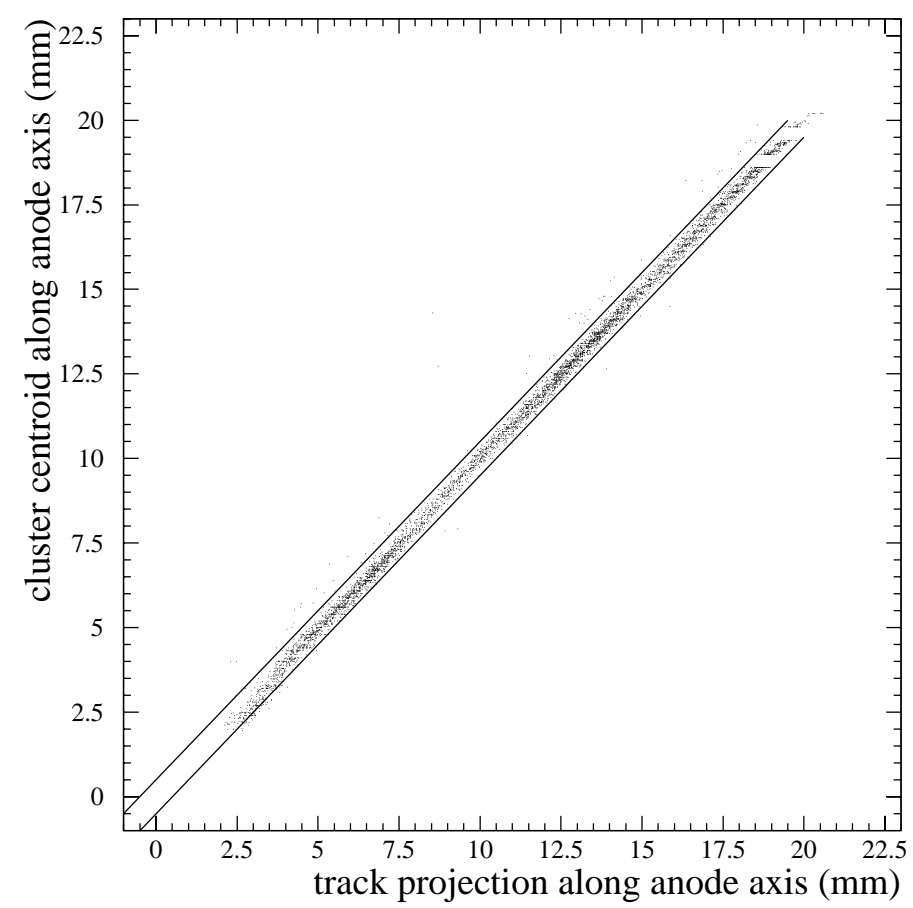

Figure 4.11. Centroid position along the anode axis versus reference position given by the microstrip telescopes. 


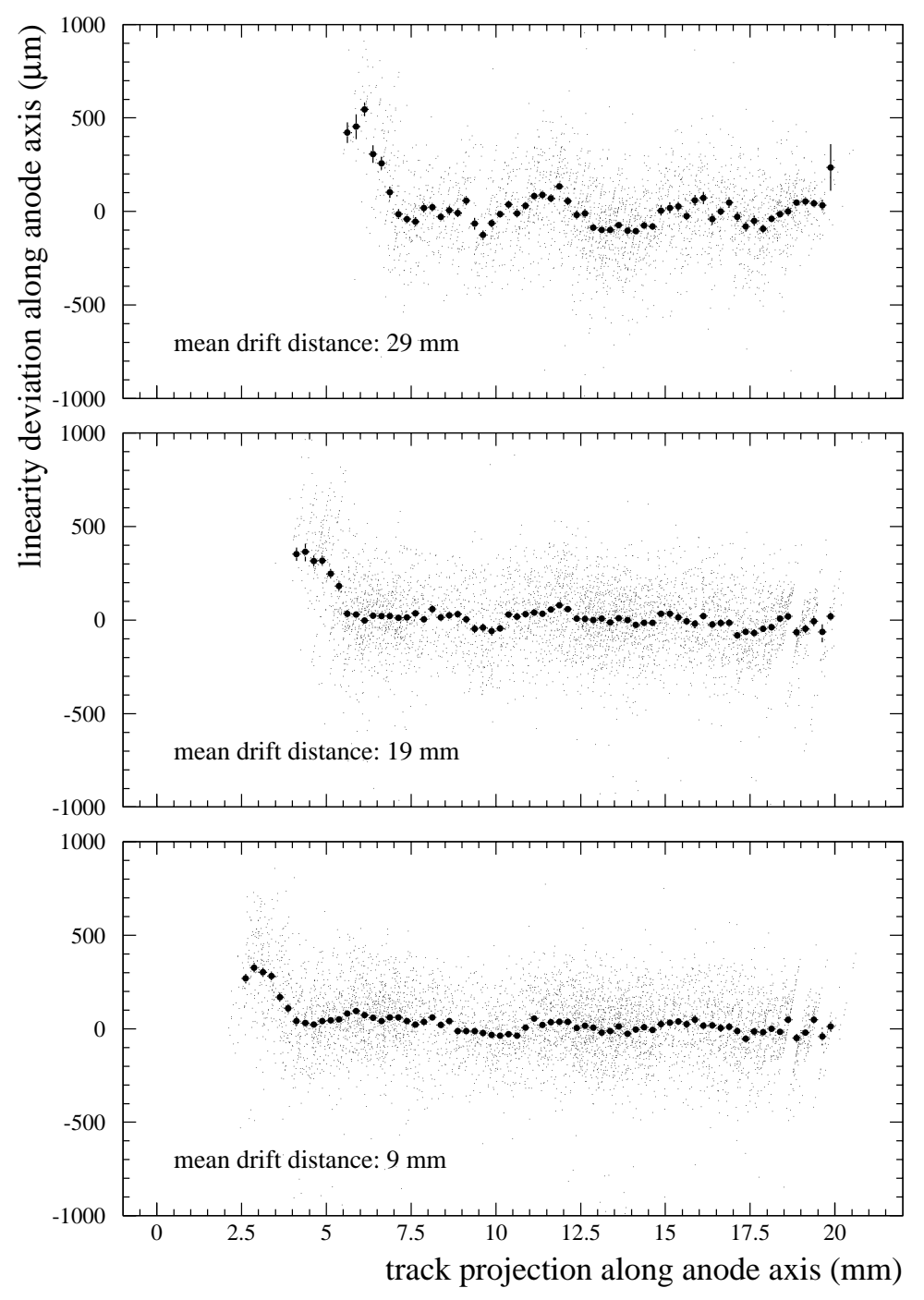

Figure 4.12. Deviation from linearity along the anode axis versus position given by the microstrip telescopes. $a$ drift distance $29 \pm 5 \mathrm{~mm}, b$ drift distance $19 \pm 5 \mathrm{~mm}, c$ drift distance $9 \pm 5 \mathrm{~mm}$ 